\title{
Legionella bozemanii, an Elusive Agent of Fatal Cavitary Pneumonia
}

\author{
A. Widmer, P. Hohl, S. Dirnhofer, S. Bassetti, S. Marsch, R. Frei
}

\begin{abstract}
A 67-year-old patient died of Legionella bozemanii pneumonia with negative urinary antigen and negative serology. Cystic lesions in pneumonia of unknown origin should lead to the differential diagnosis of $L$. bozemanii infections.
\end{abstract}

Infection 2007; 35: 180-181

DOI 10.1007/s15010-007-6251-4

A 67-year-old woman was admitted to the medical intensive care unit (ICU) for progressive acute respiratory distress with cardiac and renal failure and suspected pneumonia. On admission, her temperature was $37.6^{\circ} \mathrm{C}$, white blood count (WBC) $10.8 \times 10^{9} / 1$, C-reactive protein $(\mathrm{CRP})$ $57 \mathrm{mg} / \mathrm{l}$, and creatinine $350 \mu \mathrm{mol} / \mathrm{l}$. A chest X-ray revealed bilateral infiltrates of the lung, pleural effusions, and cardiomegaly. Electrocardiogram, cardiac enzymes and troponin levels were unremarkable.

Since 1980, she suffered from biopsy-proven mediastinal sarcoidosis. In addition, she had a 9-year history of systemic lupus erythematosus (SLE) with progressive renal failure despite prednisone and nivaquin treatment. In January 2002, a CT scan of the lung was performed due to weight loss of $16 \mathrm{~kg}$ within the last 6 months showing small cystic lesions in the left lower lobe. Her medications included amlodipinum and losartan.

She was admitted to the hospital for cardiovascular support and was treated with ceftriaxone and clarithromycin for suspected pneumonia. However, microbiological investigation including cultures of sputum, blood, urine and pleural fluid were negative. Clarithromycin was discontinued after clinical improvement and a negative urinary antigen test for Legionella pneumophila $\left(\mathrm{NOW}^{\circledR}\right.$ Legionella Urinary Antigen Test, Binax, Inc., Scarborough, ME, USA). Two days later, the pulmonary function continued to decrease, and corticosteroids were added for suspected reactivation of sarcoidosis and/or SLE.

Ceftriaxone was replaced after 12 days by piperacillin/tazobactam $(\mathrm{P} / \mathrm{T})$ for suspected superinfection dur- ing ICU stay. Despite broad-spectrum antimicrobial and corticosteroid therapy, clinical signs and symptoms deteriorated, CRP and WBC rose, and thrombocytes dropped. One day later, the patient required emergency intubation because of acute respiratory distress syndrome (ARDS). CRP was $325 \mathrm{mg} / \mathrm{l}$, WBC $18 \times 10^{9} / 1$. A CT scan continued to reveal progressing infiltrates in both lungs. A bronchial aspirate grew one colony of Aspergillus fumigatus that was interpreted as contamination. Antibiotics were switched from $\mathrm{P} / \mathrm{T}$ to meropenem due to continuous deterioration of the clinical status. Severe thrombocytopenia of unknown etiology delayed lung biopsy for 4 weeks, which demonstrated interstitial fibrosing pneumonia with accumulation of intra-alveolar foamy macrophages. Few colonies of A. fumigatus grew from biopsy material suggesting pulmonary invasive aspergillosis. Therefore, liposomal amphotericin B was added. Despite broad-spectrum antimicrobial therapy, respiratory failure developed. On repeat chest Xray and CT scan, new cavitary lesions became prominent in the right upper lobe. Because the histology of the lung did not show any signs of invasive aspergillosis, cyclosposphamid was added for possible necrotising pulmonary vasculitis. A repeated urinary antigen test and a serology for Legionella pneumophila (Legionella pneumophila [1-8] IFA, Meridian Bioscience, Cincinnati, OH, USA) remained negative. However, L. bozemanii grew from cultures of the lung biopsy and Dieterle stain demonstrated intra- and extracellular rods (Figure 1). Clarithromycin was reestablished, but the patient deceased 2 days later after start of

\footnotetext{
A. Widmer (corresponding author), P. Hohl, S. Dirnhofer, S. Bassetti, S. Marsch, R. Frei

Division of Infectious Diseases and Hospital Epidemiology, University Hospital Basel, 4031 Basel, Switzerland; Phone (+41/61) 2653-851, Fax-854, e-mail: awidmer@uhbs.ch

Peter Hohl is deceased
}

Received: September 10, 2006 • Revision accepted: February 26, 2007 


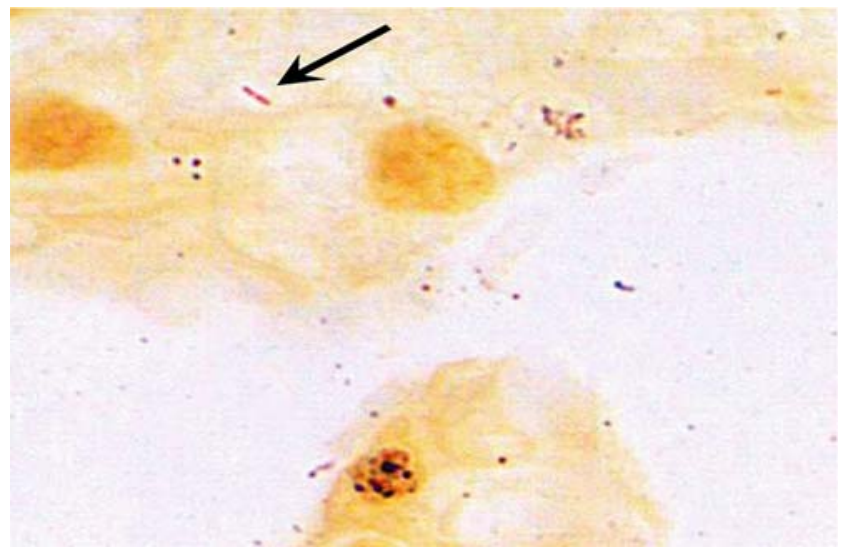

Figure 1. Section of the open lung biopsy (left lower lobe) showing alveolar foam cells and macrophages with slender intra- and extracellular rods (arrows ). (Dieterle stain; magnification 630x).

treatment. Identification of the strain was confirmed by the Swiss Legionella reference laboratory. The source of L. bozemanii remained unknown: samples from hospital faucets and from the shower and faucets of patient's home proved negative for Legionella spp.

L. bozemanii is an infrequent cause of legionellosis, even though endemic in water and soil, also in Europe. L. bozemanii infections in immunocompromised patients typically produce cavitary lung lesions $[3,4]$.

Immunocompromised patients with cystic lesions in the upper lobes frequently suffer from tuberculosis, but other diagnoses should be considered such invasive aspergillosis or an autoimmune disease [5]. Clinicians should be aware on recent reports from patients after transplantation or AIDS with L. bozemanii infections [3, 6]. Diagnosis of L. bozemanii is difficult: commonly used urinary antigen tests for Legionella and serological kits fail to consistently detect L. bozemanii [7]. The urinary antigen for Legionella is targeted against L. pneumophila serotype 1, and was repetitively negative in the present case, challenging a recent report [8]. None of the commercially available serological kits are able to identify antibodies to L. bozemanii with high sensitivity and remained negative in this case. Even microbiologic cultures using media for Legionella may be false negative since most culture media for Legionella contain cefamandole, a drug being active against $L$. bozemanii in vitro [9] in contrast to other species of Legionella.

L. bozemanii should be included in the differential diagnosis of cystic lung lesions in the lower lobes, when more frequently observed diagnoses such as tuberculosis and non-infectious diseases have been ruled out [10]. Appropriate specimens with specific information to the microbiology laboratory are crucial to isolate L. bozemanii. Lung biopsies should be investigated for Legionella e.g., by the Dieterle stain in cystic lesions of unknown origin. Today, antimicrobial treatment with a quinolone may be preferred to macrolides in the immunocompromised host $[5,11,12]$. In conclusion, clinical awareness of this rare pathogen and an interdisciplinary approach to immunocompromised patients presenting with cavitary pneumonia of unknown origin are crucial for guided treatment to decrease mortality of this severe disease. Diagnosis of L. pneumophila serogroup 1 has been facilitated by the rapid urinary antigen test. However, only cultures for Legionella are able to detect other Legionella species and should not be abandoned, particularly in severely ill patients.

\section{References}

1. Bassetti S, Widmer AF: Legionella resources on the world wide web. Clin Infect Dis 2002; 34: 1633-1640.

2. Mitchell RG, Pasvol G, Newnham RS: Pneumonia due to Legionella bozemanii: first report of a case in Europe. J Infect 1984; 8: 251-255.

3. Harris A, Lally $M$, Albrecht $M$ : Legionella bozemanii pneumonia in three patients with AIDS. Clin Infect Dis 1998; 27: 97-99.

4. Strampfer MJ, Schoch PE, Scoma S, Cunha BA: Empyema and Legionella bozemanii. Ann Intern Med 1986; 105: 626.

5. Taylor TH, Albrecht MA: Legionella bozemanii cavitary pneumonia poorly responsive to erythromycin: case report and review. Clin Infect Dis 1995; 20: 329-334.

6. Muder RR, Stout JE, Yu VL: Nosocomial Legionella micdadei infection in transplant patients: fortune favors the prepared mind. Am J Med 2000; 108: 346-348.

7. Muder RR, Yu VL: Infection due to Legionella species other than L. pneumophila. Clin Infect Dis 2002; 35: 990-998.

8. Dominguez JA, Gali N, Pedroso P, Fargas A, Padilla E, Manterola JM et al: Comparison of the Binax Legionella urinary antigen enzyme immunoassay (EIA) with the Biotest Legionella Urin antigen EIA for detection of Legionella antigen in both concentrated and non-concentrated urine samples. J Clin Microbiol 1998; 36 : 2718-2722.

9. Lee TC, Vickers RM, Yu VL, Wagener MM: Growth of 28 Legionella species on selective culture media: a comparative study. J Clin Microbiol 1993; 31: 2764-2768.

10. Yu VL, Plouffe JF, Pastoris MC, Stout JE, Schousboe M, Widmer A et al: Distribution of Legionella species and serogroups isolated by culture in patients with sporadic community-acquired legionellosis: an international collaborative survey. J Infect Dis 2002; 186: 127-128.

11. Widmer AF: Legionellosis. Ther Umsch 2001; 58: 592-598.

12. Pedro-Botet L, Yu VL: Legionella: macrolides or quinolones? Clin Microbiol Infect 2006; 12 (Suppl 3): 25-30. 